Article

\title{
Antimicrobial Susceptibility and Association with Toxin Determinants in Clostridium perfringens Isolates from Chickens
}

\author{
Bai Wei ${ }^{1,+}$, Se-Yeoun Cha ${ }^{1,+}$, Jun-Feng Zhang ${ }^{1}$, Ke Shang ${ }^{1}$, Hae-Chul Park ${ }^{2}$, \\ JeongWoo Kang ${ }^{2}{ }^{\mathbb{D}}$, Kwang-Jick Lee ${ }^{2}$, Min Kang ${ }^{1, *}$ and Hyung-Kwan Jang ${ }^{1, *}$ \\ 1 Center for Poultry Diseases Control, Department of Veterinary Infectious Diseases and Avian Diseases, \\ College of Veterinary Medicine and Jeonbuk National University, Iksan 54596, Korea; \\ weibai116@hotmail.com (B.W.); kshmnk@hanmail.net (S.-Y.C.); jfzhang018@gmail.com (J.-F.Z.) \\ shangke0624@gmail.com (K.S.) \\ 2 Veterinary Drugs \& Biologics Division, Animal and Plant Quarantine Agency (QIA), Gimcheon 39660, Korea; \\ sungpark@korea.kr (H.-C.P.); hijach@korea.kr (J.K.); leekwj@korea.kr (K.-J.L.) \\ * Correspondence: minkang@jbnu.ac.kr (M.K.); hkjang@jbnu.ac.kr (H.-K.J.); Tel.: +82-63-850-0690 (M.K.); \\ +82-63-850-0945 (H.-K.J.); Fax: +82-858-0686 (M.K.); +82-858-9155 (H.-K.J.) \\ + These authors contributed equally to this study.
}

Received: 25 October 2020; Accepted: 17 November 2020; Published: 19 November 2020

\begin{abstract}
The aim of the present study was to investigate variation in antimicrobial resistance in Clostridium perfringens (C. perfringens) isolated from chickens after withdrawal of antimicrobial growth promoters (AGPs); and to investigate the correlation between the presence of toxin genes (cpb2, netB, and tpeL) and antimicrobial resistance. Altogether, 162 isolates of $C$. perfringens were obtained from chickens displaying clinical signs of necrotic enteritis $(n=65)$ and from healthy chickens $(n=97)$ in Korea during 2010-2016. Compared to before AGP withdrawal, increased antimicrobial resistance or $\mathrm{MIC}_{50} / \mathrm{MIC}_{90}$ value was observed for nine antimicrobials including penicillin, tetracycline, tylosin, erythromycin, florfenicol, enrofloxacin, monensin, salinomycin, and maduramycin. Significantly $(p<0.05)$ higher resistance to gentamicin, clindamycin, and virginiamycin was found in isolates from chickens with necrotic enteritis compared to those from healthy chickens. tpeL gene was not detected in C. perfringens isolates from healthy chickens. A correlation between toxin gene prevalence and antibiotic resistance was found in the $C$. perfringens isolates. Because the usage of antimicrobials may contribute to the selection of both resistance and toxin genes, these can potentially make it challenging to control antimicrobial resistance in pathogenic colonies. Therefore, a more complete understanding of the interplay between resistance and virulence genes is required.
\end{abstract}

Keywords: C. perfringens; chicken; antimicrobials resistance; growth promoters; virulence factors

\section{Introduction}

Clostridium perfringens is a Gram-positive, anaerobic spore-forming bacterium that causes a wide variety of diseases in humans and animals and is ubiquitous in the environment including wastewater, dust, air, and healthy human and animal intestinal tracts [1]. Necrotic enteritis (NE) caused by C. perfringens has been one of the most economically important and financially crippling enteric poultry diseases in broiler chickens around the world [1]. From a financial point of view, the cost of subclinical NE can be as high as five cents per bird, and NE outbreaks can cost the global broiler industry nearly two billion US dollars every year [2]. Historically, C. perfringens isolates are categorized into the five toxin types A, B, C, $\mathrm{D}$, and $\mathrm{E}$, depending on the production of four major toxins including alpha $(\alpha)$, beta $(\beta)$, epsilon $(\varepsilon)$, and iota ( $\iota$. C. perfringens type $A$ is the most common toxin type isolated from chickens suffering from 
NE [1]. Recently, based on a new introduced toxin-based typing system, C. perfringens type F consists of isolates that produce $C$. perfringens enterotoxin (CPE), type $\mathrm{G}$ isolates that produce NetB toxin (encoded by the gene $n e t B$ ) which has been found to be a critical virulence factor in the pathogenicity of NE in chickens [3]. In addition to these toxin genes, tpeL, has recently been identified in some chickens with NE -derived C. perfringens [4]. Following its first description, the gene $c p b 2$ has been found in C. perfringens isolates from a variety of animals suffering from enteric disease [5].

In most modern broiler-producing countries including Korea, antimicrobial growth promoters (AGPs) have been largely used during poultry production. Considering the emergence and spread of antimicrobial resistance, Korea prohibited the addition of AGPs to animal feeds since July 2011; while anticoccidial drugs including monensin, salinomycin, and maduramycin were still used in feed additives in poultry [6,7]. Nevertheless, NE re-emerged in broiler chickens in Korea as a consequence of AGP withdrawal from feed, and a high use of antimicrobials to treat this disease in broilers has been reported [8]. In view of the above, and the fact that antimicrobial therapy is still the most effective measure to control NE, we investigated the effect of AGP withdrawal from feed on the susceptibility of C. perfringens to antimicrobials of relevance to poultry production.

Antimicrobial use is not only associated with increased antimicrobial resistance among bacterial pathogens, but selection due to antimicrobial use may also contribute to positive/negative correlation with virulence determinants [9]. Resistance and virulence may not always be independent properties, and their relationship may play an important role in the pathogenesis of C. perfringens infection [9-11]. There have been no studies demonstrating such a link between antimicrobial resistance and virulence determinants in C. perfringens populations. Therefore, the aim of the present study was to investigate the variation in antimicrobial resistance in C. perfringens isolated from chicken, especially after AGP withdrawal; we also aimed to investigate the correlation between the presence of toxin genes (cpb2, $n e t B$, and tpeL) and antimicrobial resistance in $C$. perfringens isolates.

\section{Materials and Methods}

\subsection{Bacterial Isolates}

This study was a part of the research project "Development of therapeutic technique for using alternatives to antibiotics in necrotic enteritis of broiler chickens (PJ907105)" in Korea. Altogether, 162 isolates of $C$. perfringens were obtained from chickens displaying clinical signs of NE $(n=65)$ and from healthy chickens $(n=97)$ from 2010 to 2016 in Korea. Of these, 65 isolates (24 isolates, 2010-2011; 41 isolates, 2012-2016) of C. perfringens were collected from diseased chickens with NE submitted to our lab for necropsy examination. The chickens were presented with clinical signs including bloody or dark diarrhea, severe depression, and anorexia. The tissue samples were collected under the supervision of veterinarians. The collection of samples from chicken does not require an ethics statement and the owners provided informed consent for the research and publication of the results. After dissection, the gross lesions included multiple foci of mucosal necrosis on the lower part of the small intestine in diseased chickens. C. perfringens isolates were collected from the small intestine or liver with NE. For each farm, at least 3 to 4 colonies were collected for next molecular diagnosis, and one isolate was collected from a single chicken farm after final identification. Meanwhile, isolation of C. perfringens in healthy chickens was carried out from 2010. At the beginning of the study, no permission was required for disease monitoring and the animals could be used for research or teaching purposes. The owners provided informed consent for the clinical research and all the procedure under the supervision of veterinarians. The other 97 isolates (37 isolates, 2010-2011; 60 isolates, 2012-2016) were collected from the cecum or small intestine of clinically-healthy chickens obtained from 177 farms, and one isolate was obtained per farm. Primary isolates were checked visually for a typical C. perfringens double-hemolysis zone surrounding the colonies on sheep's blood agar (Komed, Seongnam, South Korea) and then the C. perfringens isolates were confirmed by PCR reaction to detect the cpa gene [12]. Isolates were 
preserved in preservation media with Brain heart infusion (BHI, BD Becton Dickinson, Franklin Lakes, MD, USA) and $20 \%$ glycerol, and kept at $-70{ }^{\circ} \mathrm{C}$ until use.

\subsection{Antimicrobial Susceptibility Test}

Minimal inhibitory concentrations (MICs) were determined on Mueller Hinton agar plates (Oxoid Ltd., Basingstoke, England, UK) containing doubling dilutions of the antimicrobials including penicillin, ampicillin, amoxicillin, gentamicin, spectinomycin, tetracycline, tylosin, erythromycin, florfenicol, bacitracin, enrofloxacin, clindamycin, virginiamycin, monensin, salinomycin, maduramycin, and trimethoprimsulfamethoxazole, which are used for C. perfringens and/or coccidiosis infection and are widely used in poultry industry (Table 1). C. perfringens ATCC 13124 was used as the quality control strain and MIC was read as the lowest antimicrobial concentration inhibiting visible bacterial growth after 18-24 $\mathrm{h}$ of anaerobic incubation. The $\mathrm{MIC}_{50}$ and $\mathrm{MIC}_{90}$ represent the MIC value at which $\geq 50 \%$ and $\geq 90 \%$, respectively, of the isolates within a test population were inhibited.

Standardized guidelines to interpret MIC values for $C$. perfringens are not well established. Therefore, we used the interpretation criteria for gentamicin and trimethoprim-sulfamethoxazole available from the Clinical and Laboratory Standards Institute (CLSI) for antimicrobial susceptibility testing of anaerobic bacteria [13], and those for penicillin, ampicillin, clindamycin [14], amoxicillin [15], enrofloxacin, tetracycline, florfenicol, bacitracin, erythromycin, and virginiamycin from published literature [16,17].

\subsection{Toxin Gene Detection}

All C. perfringens isolates were tested for the presence of $c p a, c p b, i A$, etx, $c p b 2$, and $c p e$ which respectively encode alpha toxin, beta toxin, iota toxin, epsilon toxin, beta2 toxin, and enterotoxin of $C$. perfringens, based on a procedure described previously [12]. C. perfringens ATCC 13124 was as positive control for cpa used in this study. The presence of the netB and tpeL genes was detected based on a procedure described previously [18]. Electrophoresis was performed on a $1 \%$ agarose gel with ethidium bromide using standard procedures.

\subsection{Statistical analyses}

Data management and all statistical analyses were done using the software SPSS version 19.0 (IBM, Armonk, NY, USA). The Chi-Square test was used to compare groups for the difference between antimicrobial resistance and prevalence of toxin genes. Association between various antimicrobial resistance outcomes (resistance or susceptibility) and presence of the toxin genes was determined via calculation of odds ratios (OR) with $95 \%$ confidence intervals (CI). An OR of $>1$ indicated that toxin gene prevalence was positively associated with resistance, and an OR of $<1$ indicated a negative association. All statistical significance tests were done using a cut-off value of $5 \%$. 
Table 1. Minimal inhibitory concentrations (MICs) against Clostridium perfringens isolates from chickens from 2010 to 2016.

\begin{tabular}{|c|c|c|c|c|c|c|c|c|c|}
\hline \multirow{2}{*}{ Antimicrobial } & \multirow{2}{*}{ Range } & \multirow{2}{*}{$\begin{array}{c}\text { Breakpoint } \\
(\mu \mathrm{g} / \mathrm{mL})\end{array}$} & \multicolumn{2}{|c|}{ Total $(n=162)$} & \multicolumn{2}{|c|}{$2010-2011(n=61)$} & \multicolumn{2}{|c|}{$2012-2016(n=101)$} & \multirow{2}{*}{$p$ Value } \\
\hline & & & $\mathrm{MIC}_{50} / \mathrm{MIC}_{90}$ & Resistance No. (\%) & $\mathrm{MIC}_{50} / \mathrm{MIC}_{90}$ & Resistance No. (\%) & $\mathrm{MIC}_{50} / \mathrm{MIC}_{90}$ & Resistance No. (\%) & \\
\hline Penicillin & $\leq 0.06$ to 2 & 1 & $\leq 0.06 / 0.25$ & $2(1.2)$ & $\leq 0.06 / 0.12$ & 0 & $\leq 0.06 / 0.25$ & $2(2.0)$ & \\
\hline Ampicillin & $\leq 0.12$ to 0.25 & 16 & $\leq 0.12 / 0.25$ & 0 & $\leq 0.12 / 0.25$ & 0 & $\leq 0.12 / \leq 0.12$ & 0 & \\
\hline Amoxicillin & $\leq 0.25$ & 16 & $\leq 0.25 / \leq 0.25$ & 0 & $\leq 0.25 / \leq 0.25$ & 0 & $\leq 0.25 / \leq 0.25$ & 0 & \\
\hline Gentamicin & 4 to $\geq 16$ & 16 & $\geq 16 / \geq 16$ & $151(93.2)$ & $\geq 16 / \geq 16$ & $61(100.0)$ & $\geq 16 / \geq 16$ & $90(89.1)$ & 0.007 \\
\hline Spectinomycin & 8 to $\geq 128$ & -b & $\geq 128 / \geq 128$ & -b & $\geq 128 / \geq 128$ & -b & $\geq 128 / \geq 128$ & -b & $-b$ \\
\hline Tetracycline & 0.25 to $\geq 16$ & 4 & $4 / \geq 16$ & $98(60.5)$ & $4 / 8$ & $32(52.5)$ & $8 / \geq 16$ & $66(65.3)$ & \\
\hline Tylosin & $\leq 2$ to $\geq 16$ & $-b$ & $\leq 2 / \geq 16$ & b & $\leq 2 / 8$ & b & $\leq 2 / 16$ & b & $-b$ \\
\hline Erythromycin & 0.12 to $\geq 16$ & 16 & $4 / \geq 16$ & $37(22.8)$ & $2 / \geq 16$ & $16(26.2)$ & $4 / \geq 16$ & $21(20.8)$ & \\
\hline Florfenicol & 1 to 8 & 8 & $1 / 2$ & $1(0.6)$ & $\leq 1 / \leq 1$ & 0 & $2 / 2$ & $1(1.0)$ & \\
\hline Bacitracin & 1 to 64 & 32 & $1 / 32$ & $28(17.3)$ & $\leq 1 / 32$ & $9(14.8)$ & $\leq 1 / 32$ & $19(18.8)$ & \\
\hline Enrofloxacin & 0.12 to $\geq 32$ & 2 & $1 / \geq 32$ & $71(43.8)$ & $0.25 / 32$ & $15(24.6)$ & $2 / 32$ & $56(55.4)$ & $<0.001$ \\
\hline Clindamycin & 0.5 to $\geq 8$ & 8 & $2 / \geq 8$ & $62(38.3)$ & $\geq 8 / \geq 8$ & $32(52.5)$ & $2 / \geq 8$ & $30(29.7)$ & 0.004 \\
\hline Virginiamycin & 1 to 16 & 4 & $4 / 8$ & $133(82.1)$ & $4 / \geq 16$ & $60(98.4)$ & $4 / \geq 16$ & $73(72.3)$ & $<0.001$ \\
\hline Monensin & $\leq 0.12$ to 4 & $-b$ & $1 / 2$ & b & $\leq 0.12 / 0.25$ & b & $1 / 2$ & b & $-\mathrm{b}$ \\
\hline Salinomycin & $\leq 0.12$ to 0.5 & $-b$ & $0.25 / 0.25$ & $-b$ & $\leq 0.12 / \leq 0.12$ & $-b$ & $0.25 / 0.25$ & - b & - b \\
\hline Maduramycin & 0.12 to 0.5 & $-b$ & $0.25 / 0.5$ & $-b$ & $0.25 / 0.25$ & $-b$ & $0.25 / 0.5$ & $-b$ & $-b$ \\
\hline $\begin{array}{l}\text { Trimethoprim- } \\
\text { sulfamethoxazole }\end{array}$ & $0.5 / 9.5$ to $>4 / 76$ & $>4 / 76$ & $4 / 76 / 4 / 76$ & $126(77.8)$ & $\geq 4 / 76 / \geq 4 / 76$ & $44(72.1)$ & $\geq 4 / 76 / \geq 4 / 76$ & $82(81.2)$ & \\
\hline
\end{tabular}

a, The Chi-square test of independence was used to test the difference in antimicrobial resistance between Clostridium perfringens isolates from 2010-2011 and 2012-2016; $p$ value > 0.05 is not shown. $-{ }^{b}$, not available. 


\section{Results}

\subsection{Antimicrobial Susceptibility Testing}

The distribution of MICs of the 17 antimicrobial agents tested against the 162 C. perfringens isolates from chickens is shown in Table 1 and Figure S1. High prevalence of resistance was observed against gentamicin $(93.2 \%)$, virginiamycin $(82.1 \%)$, trimethoprim-sulfamethoxazole $(77.8 \%)$, and tetracycline (60.5\%). Moreover, resistance to enrofloxacin (43.8\%), clindamycin (38.3\%), erythromycin (22.8\%), bacitracin $(17.3 \%)$, penicillin $(1.2 \%)$, and florfenicol $(0.6 \%)$ was also found. All isolates showed sensitivity to ampicillin and amoxicillin and showed a low MIC of these two antimicrobials. The $\mathrm{MIC}_{50} / \mathrm{MIC}_{90}$ ratios for monensin, salinomycin, and maduramycin were $1 / 2,0.25 / 0.25$, and $0.25 / 0.5 \mu \mathrm{g} / \mathrm{mL}$, respectively.

Compared to C. perfringens isolates from 2010-2011, those from 2012-2016 were more likely to be resistant to most of the antimicrobials tested in the present study. A significantly $(p<0.05)$ higher percentage of resistance to enrofloxacin was found in C. perfringens isolates from 2012-2016. Further, the $\mathrm{MIC}_{50}$ and/or $\mathrm{MIC}_{90}$ values of penicillin, tetracycline, tylosin, erythromycin, florfenicol, enrofloxacin, monensin, salinomycin, and maduramycin against the 2012-2016 isolates were found to be higher than those against the 2010-2011 isolates. A significantly $(p<0.05)$ lower percentage of resistance to gentamicin, clindamycin, and virginiamycin was found in the 2012-2016 isolates than in the 2010-2011 isolates.

The prevalence of antimicrobial resistance in C. perfringens isolates from healthy chickens and chickens with NE differed against five antimicrobials (Table 2). Significantly $(p<0.05)$ higher resistance to gentamicin, clindamycin, and virginiamycin but significantly $(p<0.05)$ lower resistance to enrofloxacin was found in C. perfringens from chickens with NE compared with healthy chickens. There was no difference $(p>0.05)$ in antimicrobial resistance to the other five antimicrobials including penicillin, tetracycline, erythromycin, bacitracin, clindamycin, and trimethoprim-sulfamethoxazole between the isolates from healthy chickens and chickens with NE.

Table 2. Antimicrobial resistance of Clostridium perfringens isolates from chickens with or without necrotic enteritis.

\begin{tabular}{cccc}
\hline \multirow{2}{*}{ Antimicrobial } & \multicolumn{2}{c}{ Antibiotic Resistance, $\boldsymbol{n / ( \% )}$} & \multirow{2}{*}{$\boldsymbol{p}$ Value $^{\mathbf{a}}$} \\
\cline { 2 - 3 } & Enteritis $(\boldsymbol{n}=\mathbf{6 5 )}$ & Health $(\boldsymbol{n = 9 7 )}$ & \\
\hline Penicillin & 0 & $2(2.1)$ & \\
Gentamicin & $65(100.0)$ & $86(88.7)$ & 0.007 \\
Tetracycline & $33(50.8)$ & $65(67.0)$ & \\
Erythromycin & $19(29.2)$ & $18(18.6)$ & \\
Bacitracin & $12(18.5)$ & $16(16.5)$ & \\
Enrofloxacin & $18(27.7)$ & $53(54.6)$ & $<0.001$ \\
Clindamycin & $35(53.8)$ & $27(27.8)$ & 0.004 \\
Virginiamycin & $64(98.5)$ & $69(71.1)$ & $<0.001$ \\
Trimethoprim-sulfamethoxazole & $48(73.8)$ & $78(80.4)$ & \\
\hline
\end{tabular}

a , The Chi-square test of independence was used to test the difference in antimicrobial resistance between Clostridium perfringens isolates from chickens with or without necrotic enteritis; $p$ value $>0.05$ is not shown.

\subsection{Toxin Gene Prevalence in C. perfringens Isolates}

Results of PCR assays used for toxinotyping of $C$. perfringens showed that all 162 isolates were found to have only the $c p a$ except for netB gene. Among these, 38 isolates with net $B$ gene were $C$. perfringens type $\mathrm{G}$ and other isolates are type A. A total of 126 (77.8\%), 38 (23.5\%), and eight (4.9\%) isolates were positive for $c p b 2$, netB, and tpeL genes, respectively, in PCR assays (Table 3). The cpb2 and netB genes were both detected in C. perfringens isolates from healthy chickens and chickens with NE, and the tpeL gene was only detected in C. perfringens isolates from chickens with NE. Significantly $(p<0.05)$ higher prevalence of the $c p b 2$, netB, and tpeL genes was found in $C$. perfringens isolates from chickens with NE $(87.7 \%, 36.9 \%$, and $12.3 \%)$ than in the isolates from healthy chickens $(71.1,14.4$, and $0 \%)$. 
Table 3. Toxin gene prevalence in Clostridium perfringens isolates from chickens with or without necrotic enteritis.

\begin{tabular}{|c|c|c|c|c|c|c|c|c|c|}
\hline & \multirow{2}{*}{$\begin{array}{l}\text { No. of } \\
\text { Isolates }\end{array}$} & \multicolumn{3}{|c|}{ No. $(\%)$ of Toxin Genes } & \multicolumn{5}{|c|}{ Toxin Gene Pattern, $n /(\%)$} \\
\hline & & $c p b 2$ & netB & tpeL & $c p b 2 / n e t B / t p e L$ & $c p b 2 / n e t B$ & $c p b 2$ & netB & No Toxin Gene \\
\hline Total & 162 & $\begin{array}{c}126 \\
(77.8)\end{array}$ & $38(23.5)$ & $8(4.9)$ & $8(4.9)$ & $28(17.3)$ & $90(55.6)$ & $2(1.2)$ & $34(21.0)$ \\
\hline Enteritis & 65 & $57(87.7)$ & $24(36.9)$ & $8(12.3)$ & $8(12.3)$ & $15(23.1)$ & $34(52.3)$ & $1(1.5)$ & $7(10.8)$ \\
\hline Healthy & 97 & 69 (71.1) & 14 (14.4) & $0(0.0)$ & 0 & 13 (13.4) & $56(57.7)$ & $1(1.0)$ & $27(27.8)$ \\
\hline$p$ value $^{\mathrm{a}}$ & & 0.013 & 0.001 & 0.001 & 0.001 & & & & 0.009 \\
\hline
\end{tabular}

${ }^{a}$, The Chi-square test of independence was used to test the difference in toxin gene prevalence between Clostridium perfringens isolates from chickens with or without necrotic enteritis; $p$ value $>0.05$ is not shown.

A total of four toxin gene patterns including the presence of $c p b 2 / n e t B / t p e L, c p b 2 / n e t B, c p b 2$, or netB were found in the 162 C. perfringens isolates (Table 3), and the toxin gene pattern cpb2/netB/tpeL was only found in the isolates from chickens with NE. A total of 34 isolates with no toxin genes $(c p b 2$, netB, and tpeL) was detected in the present study, and a significantly $(p<0.05)$ higher prevalence of these isolates was found in healthy chickens $(27.8 \%)$ than in chickens with NE $(10.8 \%)$.

\subsection{Associations between Antimicrobial Resistance Outcomes and the Presence of Toxin Genes}

The relationship between antimicrobial resistance and toxin gene carriage is shown in Table 4 . Carriage of $c p b 2$ was more common among isolates resistant to gentamicin (OR, 2.222; 95\% CI, 1.013-4.874; $p=0.001)$, tetracycline (OR, 1.512; 95\% CI, 1.222-1.872; $p<0.001)$, or trimethoprim-sulfamethoxazole (OR, $1.351 ; 95 \% \mathrm{CI}, 1.028-1.774 ; p=0.006)$, while being more common among bacitracin-susceptible isolates (OR, $0.696 ; 95 \% \mathrm{CI}, 0.5-0.969 ; p=0.004)$. The netB gene was more common among virginiamycin-resistant isolates (OR, 8.068; 95\% CI, 1.153-56.436; $p=0.005)$ than virginiamycin-susceptible isolates, while being more common among those susceptible to tetracycline (OR, 0.266; 95\% CI, $0.142-0.498 ; p<0.001$ ), erythromycin (OR, 0.29; 95\% CI, 0.094-0.888; $p=0.012)$, enrofloxacin (OR, 0.458; 95\% CI, 0.239-0.879; $p=0.013$ ), or clindamycin (OR, $0.501 ; 95 \% \mathrm{CI}, 0.254-0.986 ; p=0.034)$ than in the corresponding resistant isolates. The tpeL gene was more common among isolates susceptible to tetracycline (OR, $0.218,95 \% \mathrm{CI}$, $0.045-1.045 ; p=0.035)$ or enrofloxacin $(\mathrm{OR}, 0.142 ; 95 \% \mathrm{CI}, 0.018-1.092 ; p=0.035)$ than in the corresponding resistant isolates. 
Table 4. Associations between antimicrobial resistance outcomes and the presence of toxin genes in C. perfringens isolates from chickens.

\begin{tabular}{|c|c|c|c|c|c|c|c|c|c|c|c|c|}
\hline \multirow{3}{*}{ Antimicrobial } & \multicolumn{12}{|c|}{$\%$ of the Isolates Positive for Toxin Genes ${ }^{a}$} \\
\hline & \multicolumn{4}{|c|}{$c p b 2$} & \multicolumn{4}{|c|}{ netB } & \multicolumn{4}{|c|}{ tpeL } \\
\hline & $\mathbf{R}$ & S & OR $(95 \% \mathrm{CI})$ & $p$ Value ${ }^{\mathrm{b}}$ & $\mathbf{R}$ & $\mathrm{S}$ & OR $(95 \% \mathrm{CI})$ & $p$ Value $^{\mathrm{b}}$ & $\mathbf{R}$ & $\mathrm{S}$ & OR $(95 \% \mathrm{CI})$ & $p$ Value $^{\mathrm{b}}$ \\
\hline Gentamicin & 80.8 & 36.4 & $2.222(1.013-4.874)$ & 0.001 & 25.2 & 0 & $3.314(0.494-22.210)$ & & 5.3 & 0 & $0.765(0.105-5.577)$ & \\
\hline Tetracycline & 89.8 & 59.4 & $1.512(1.222-1.872)$ & $<0.001$ & 11.2 & 42.2 & $0.266(0.142-0.498)$ & $<0.001$ & 2.0 & 9.4 & $0.218(0.045-1.045)$ & 0.035 \\
\hline Erythromycin & 70.3 & 80.0 & $0.878(0.7-1.102)$ & & 8.1 & 28.0 & $0.29(0.094-0.888)$ & 0.012 & 0 & 6.4 & $0.362(0.047-2.768)$ & \\
\hline Bacitracin & 57.1 & 82.1 & $0.696(0.5-0.969)$ & 0.004 & 10.7 & 26.1 & $0.41(0.136-1.24)$ & & 0 & 6 & $0.504(0.066-3.827)$ & \\
\hline Enrofloxacin & 77.5 & 78.0 & $0.993(0.841-1.172)$ & & 14.1 & 30.8 & $0.458(0.239-0.879)$ & 0.013 & 0.0 & 8.8 & $0.142(0.018-1.092)$ & 0.01 \\
\hline Clindamycin & 79.0 & 77.0 & $1.026(0.868-1.213)$ & & 14.5 & 29.0 & $0.501(0.254-0.986)$ & 0.034 & 3.2 & 6.0 & $0.538(0.112-2.581)$ & \\
\hline Virginiamycin & 80.5 & 65.5 & $1.228(0.931-1.62)$ & & 27.8 & 3.4 & $8.068(1.153-56.436)$ & 0.005 & 6.0 & 0 & $2.067(0.272-15.716)$ & \\
\hline $\begin{array}{l}\text { Trimethoprim- } \\
\text { sulfamethoxazole }\end{array}$ & 82.5 & 61.1 & $1.351(1.028-1.774)$ & 0.006 & 25.4 & 16.7 & $1.524(0.692-3.355)$ & & 3.2 & 11.1 & $0.286(0.075-1.086)$ & \\
\hline
\end{tabular}

a , R, resistance; S, susceptible; OR, odd ratios; $\mathrm{CI}$, confidence intervals. ${ }^{\mathrm{b}}$, The Chi-square test of independence was used to test the difference in toxin gene prevalence between Clostridium perfringens isolates with or without antibiotic resistance; $p$ value $>0.05$ is not shown. 


\section{Discussion}

In the present study, we investigated antimicrobial resistance in C. perfringens isolates from chickens in Korea. Among all the tested antimicrobials, penicillin, ampicillin, amoxicillin, florfenicol, monensin, salinomycin, and maduramycin had relatively low MIC values, in agreement with studies conducted in China, Canada, and the United States which reported that these antimicrobials were effective in treating NE in chickens $[16,19,20]$. We also investigated the antimicrobial resistance of C. perfringens isolates from chickens before and after AGP withdrawal in Korea. It should be emphasized that AGP withdrawal from feed did not result in a comprehensive decrease in resistance; a higher level of resistance to nine antimicrobials as reflected in their $\mathrm{MIC}_{50}$ and/or $\mathrm{MIC}_{90}$ values (penicillin, tetracycline, tylosin, erythromycin, florfenicol, enrofloxacin, monensin, salinomycin, and maduramycin) was found in C. perfringens isolates obtained after AGP withdrawal compared with those obtained before (Table 1). These results most likely reflect the fact that rather than antimicrobials supplied in animal feed, much larger amounts of antimicrobials had been used for disease prophylaxis and treatment in poultry production [21]. Remarkably, the total sale of antimicrobials in Korea decreased to its lowest level in 2013, while it increased again from 2014 (Animal and Plant Quarantine Agency, 2017). Therefore, the termination of the use of AGPs in animal feed might not significantly influence antimicrobial resistance over the several subsequent years (Tables S1 and S2), and co-selection due to other antimicrobials or resistant strains with low/no fitness cost will promote the persistence of resistance in farm animals [22-24].

Compared to the antimicrobial resistance in C. perfringens from healthy chickens, significantly higher resistance to gentamicin, clindamycin, and virginiamycin was found in chickens with NE. Among these three antimicrobials, virginiamycin is the one considered for control of C. perfringens in chickens [16]. Our finding is in agreement with previous studies showing that chickens with NE have a higher probability of being exposed to antimicrobial therapy than clinically healthy chickens, and that C. perfringens isolated from diseased chickens have a higher probability of developing resistance [20]. Therefore, the C. perfringens isolates with higher resistance found in chickens with NE suggest that a more cautious approach is required in selecting proper antimicrobials for disease treatment, and to prevent emergence and dissemination of resistant C. perfringens.

All of the isolates in the present study were classified as C. perfringens type A and G, and this finding was consistent with those reported previously in Korea and other countries, which indicated that type $A$ and $G$ is prevalent both in healthy and diseased chickens $[1,3,18]$. It has recently been reported that a high frequency of toxin genes was found in C. perfringens isolates from chickens with NE than in those from healthy chickens [25]. Our finding was also consistent with the above report, with all three toxin genes tested in this study showing high prevalence in NE -derived C. perfringens isolates. We also showed that $27.8 \%$ of healthy chicken isolates did not carry any toxin genes, which were significantly $(p<0.05)$ more than the corresponding NE-derived isolates $(10.8 \%)$. This strongly supports a correlation between the presence of these toxin genes and NE, and the fact that these toxin genes play an important role in the pathogenesis of NE in chickens $[1,25]$. Further, the toxin gene tpeL and the toxin gene pattern $c p b 2 / n e t B / t p e L$ were only found in isolates from chickens with NE and not in the healthy chicken isolates. This finding strongly indicated the involvement of the toxin gene tpeL in pathogenesis of NE in chickens as previously described [4]. The tpeL gene may potentiate the effect of other virulence attributes of NE -derived C. perfringens isolates as previously reported $[4,26]$, and all the isolates from chickens with NE carrying the tpeL gene were found to co-carry $c p b 2 / n e t B$ in the present study. As the contribution of $c p b 2$ and tpeL toxin genes in the pathogenesis of NE in chickens is yet to be established. Therefore, the contribution of $c p b 2$, tpeL, tpeL, and $c p b 2 /$ netB to the pathogenesis of NE needs to be evaluated further.

Our analysis of a large population of $C$. perfringens isolates from chickens provides epidemiological evidence for the association of antimicrobial resistance and the presence of toxin genes. First, net $B$ gene prevalence had lower odds of association with resistance to tetracycline, erythromycin, enrofloxacin, or clindamycin in C. perfringens isolates, and cpb2 had lower odds of association with bacitracin resistance. 
A negative correlation between the prevalence of virulence genes and antimicrobial resistance has been reported in E. coli, with quinolone resistance being negatively correlated with the prevalence of virulence genes [27]. This may be explained by the hypothesis that mutations in topoisomerase genes produce a reduction in DNA supercoiling that might have implications in the expression of some virulence genes [27]. Further, the resistance and toxin genes might be located on different incompatible plasmid groups, and co-existence of resistance and toxin genes in a strain may be uncommon [28]. Second, a positive correlation between the prevalence of toxin genes and antimicrobial resistance was found; netB gene prevalence had higher odds of association with $C$. perfringens isolates with virginiamycin resistance, and $c p b 2$ had higher odds of association with isolates resistant to gentamicin, tetracycline, or trimethoprim-sulfamethoxazole. Vilei et al. [10] have demonstrated that aminoglycoside treatment induces the expression of the $c p b 2$ gene in C. perfringens isolates obtained from horses, and that the treatment lead to a more accentuated and fatal progression of equine typhlocolitis [10]. These data provide evidence that antimicrobial selection pressure may not only result in antimicrobial resistance, but may also be involved in virulence gene expression and disease symptom status [9,29]. The continuous use of antimicrobials in animals may facilitate the spread of virulence genes within bacterial communities, and the acquisition of resistance and virulence determinants at the same time may represent a survival advantage to the microorganisms. In our study, the positive association of $n e t B$ gene prevalence with virginiamycin resistance was observed; because the net $B$ gene is a critical factor in NE development, high virginiamycin resistance and persistent usage of virginiamycin in farm animals may promote resistance and maintenance of pathogenic colonies in farms. We found that the total netB gene prevalence in C. perfringens isolates in our study (23.5\%) was higher than that in a previous report (14.9\%) in Korea [18]; these data raise the possibility that netB gene prevalence will continually increase if virginiamycin is used without limitation in farm animals in the future. Similar to the common use of virginiamycin as an antimicrobial to treat poultry disease, tetracycline and trimethoprim-sulfamethoxazole have also been largely used in the poultry industry. Such large usage of antimicrobials in animals may also promote the dissemination of $c p b 2$ in C. perfringens strains, not limited to chickens but also to other animals, although there is currently no direct evidence to support such a speculation.

Although our results suggested a plausible ecological and biological link between antimicrobial resistance outcomes and toxin gene existence in C. perfringens isolates via epidemiological evidence, the link between resistance and virulence is complex due to the diversity of antimicrobial resistance genes, virulence factors, and bacterial species. Our ability to show the linkage between antimicrobial resistance and toxin genes has limitations; we did not investigate the resistance mechanisms for all the antimicrobials due to the limited information available on antimicrobial resistance mechanisms in C. perfringens. However, our study is the first step in understanding whether there is a connection between resistance and virulence. We also did not investigate the genetic linkage, if any, between virulence and resistance genes. More in-depth molecular studies on the interactions between antimicrobial resistance and virulence determinants are needed to fully understand the interplay between resistance and virulence genes in C. perfringens.

\section{Conclusions}

In conclusion, the present study generated data regarding antimicrobial resistance and distribution of toxin genes among C. perfringens isolates from healthy and diseased chickens before and after the AGP ban in Korea, and analyzed the association between antimicrobial resistance and toxin gene prevalence. Our results show widespread decrease in susceptibility to antimicrobials in C. perfringens isolates obtained after the AGP ban, likely as a consequence of an increase in antimicrobial usage. The unique susceptibility pattern demonstrated in this study indicates potential strategies for therapeutic candidate selection for disease control after the AGP ban. High prevalence of toxin genes were found in C. perfringens isolates from diseased chickens. The toxin gene tpeL was only found in C. perfringens isolates from diseased chickens, indicating that tpeL may contribute significantly to the pathogenesis of 
$\mathrm{NE}$; all tpeL-positive isolates showed the presence of $c p b 2$ and netB, suggesting that tpeL may potentiate the effect of other toxin determinants contributing to the pathogenesis of NE. In addition, a correlation between toxin gene prevalence and antimicrobial resistance was observed in C. perfringens communities, with both positive and negative correlations between the presence of toxin genes and antimicrobial resistance. Because the usage of antimicrobials may contribute to the selection of both resistance and toxin genes, these can potentially make it challenging to control antimicrobial resistance in pathogenic colonies. Therefore, a more complete understanding of the interplay between resistance and virulence genes is required.

Supplementary Materials: The following are available online at http://www.mdpi.com/2076-2607/8/11/1825/s1, Table S1: Antimicrobials used in chickens from 2010 to 2016 in Korea. Table S2: Antimicrobials used in feed additives and disease treatment in chickens from 2010 to 2016 in Korea. Figure S1: MIC histograms for 162 Clostridium perfringens isolates from chickens against 17 antimicrobials (A-Q) from 2010 to 2016, isolates from 2010-2011 (black) and 2012-2016 (grey).

Author Contributions: B.W., M.K. and H.-K.J. contributed to conception and design of experiments. S.-Y.C. and B.W. contributed to acquisition, analysis, and interpretation of data. B.W., S.-Y.C., J.-F.Z., K.S., H.-C.P., J.K., K.-J.L., M.K., H.-K.J. drafted and/or revised the article. All authors have read and agreed to the published version of the manuscript.

Funding: This work was supported by Korea Institute of Planning and Evaluation for Technology in Food, Agriculture and Forestry (IPET) through Agriculture, Food and Rural Affairs Convergence Technologies Program for Educating Creative Global Leader (716002-7, 320005-4) and Animal Disease Management Technology Development Program (119059-2), funded by Ministry of Agriculture, Food and Rural Affairs (MAFRA). And this paper was supported by research funds for newly appointed professors of Jeonbuk National University in 2019.

Conflicts of Interest: The authors declare no conflict of interest.

\section{References}

1. Timbermont, L.; Haesebrouck, F.; Ducatelle, R.; Van Immerseel, F. Necrotic enteritis in broilers: An updated review on the pathogenesis. Avian Pathol. 2011, 40, 341-347. [CrossRef] [PubMed]

2. Keyburn, A.L.; Boyce, J.D.; Vaz, P.; Bannam, T.L.; E Ford, M.; Parker, D.; Di Rubbo, A.; Rood, J.I.; Moore, R.J. NetB, a New Toxin That Is Associated with Avian Necrotic Enteritis Caused by Clostridium perfringens. PLoS Pathog. 2008, 4. [CrossRef] [PubMed]

3. Rood, J.I.; Adams, V.; Lacey, J.; Lyras, D.; McClane, B.A.; Melville, S.B.; Moore, R.J.; Popoff, M.R.; Sarker, M.R.; Songer, J.G.; et al. Expansion of the Clostridium perfringens toxin-based typing scheme. Anaerobe 2018, 53, 5-10. [CrossRef]

4. Coursodon, C.; Glock, R.; Moore, K.; Cooper, K.; Songer, J. TpeL-producing strains of Clostridium perfringens type A are highly virulent for broiler chicks. Anaerobe 2012, 18, 117-121. [CrossRef] [PubMed]

5. Van Asten, A.J.; Nikolaou, G.N.; Gröne, A. The occurrence of cpb2-toxigenic Clostridium perfringens and the possible role of the $\beta 2$-toxin in enteric disease of domestic animals, wild animals and humans. Veter. J. 2010, 183, 135-140. [CrossRef] [PubMed]

6. Maron, D.F.; Smith, T.J.S.; Nachman, K.E. Restrictions on antimicrobial use in food animal production: An international regulatory and economic survey. Glob. Health 2013, 9, 48. [CrossRef] [PubMed]

7. Animal and Plant Quarantine Agency. Establishment of Antimicrobial Resistance Surveillance System for Livestock, 2016. Ministry of Agriculture, Food and Rural Affairs. 2017. Available online: http://www.fao.or g/fao-who-codexalimentarius/sh-proxy/en/?lnk=1\&url=https\%253A\%252F\%252Fworkspace.fao.org\%252 Fsites\%252Fcodex\%252FMeetings\%252FCX-804-05\%252FSIDE\%20EVENTS\%252FRepublic_of_Korea.pdf (accessed on 27 November 2017).

8. Abid, S.A.; Azeem, T.; Chaudhary, Z.I.; Ahmad, M.U.D.; Rehman, Z.U.; Umar, S. Emerging threat of necrotic enteritis in poultry and its control without use of antibiotics: A review. J. Anim. Plant. Sci. 2016, 26, 1556-1567.

9. Beceiro, A.; Tomás, M.; Bou, G. Antimicrobial Resistance and Virulence: A Successful or Deleterious Association in the Bacterial World? Clin. Microbiol. Rev. 2013, 26, 185-230. [CrossRef] 
10. Vilei, E.M.; Schlatter, Y.; Perreten, V.; Straub, R.; Popoff, M.R.; Gibert, M.; Grone, A.; Frey, J. Antibiotic-induced expression of a cryptic cpb2 gene in equine $\beta 2$-toxigenic Clostridium perfringens. Mol. Microbiol. 2005, 57, 1570-1581. [CrossRef]

11. Ahn, D.; Prince, A. Host-Pathogen Interface: Progress in Understanding the Pathogenesis of Infection Due to Multidrug-Resistant Bacteria in the Intensive Care Unit. J. Infect. Dis. 2017, 215, S1-S8. [CrossRef]

12. Heikinheimo, A.; Korkeala, H. Multiplex PCR assay for toxinotyping Clostridium perfringens isolates obtained from Finnish broiler chickens. Lett. Appl. Microbiol. 2005, 40, 407-411. [CrossRef] [PubMed]

13. Clinical and Laboratory Standards Institute (CLSI). Performance Standards for Antimicrobial Susceptibility Testing; Twenty-Third Informational Supplement. 2013; Volume M100-S23. Available online: https://clsi.org/m edia/2663/m100ed29_sample.pdf (accessed on 11 January 2019).

14. British Society for Antimicrobial Chemotherapy (BASC). BSAC Methods for Antimicrobial Susceptibility Testing. Available online: http://bsac.org.uk/wp-content/uploads/2012/02/BSAC-disc-susceptibility-testingmethod-Jan-2015.pdf (accessed on 14 January 2015).

15. The European Committee on Antimicrobial Susceptibility Testing, Breakpoint Tables for Interpretation of MICs and Zone Diameters, Version 7.1. 2017. Available online: https://www.eucast.org/clinical_breakpoints/ (accessed on 25 January 2020).

16. Slavic, D.; Boerlin, P.; Fabri, M.; Klotins, K.C.; Zoethout, J.K.; Weir, P.E.; Bateman, D. Antimicrobial susceptibility of Clostridium perfringens isolates of bovine, chicken, porcine, and turkey origin from Ontario. Can. J. Veter Res. Rev. Can. Rech. Veter 2011, 75, 89-97.

17. Gad, W.; Hauck, R.; Kruger, M.; Hafez, H.M. In vitro determination of antibiotic sensitivities of Clostridium perfringens isolates from layer flocks in Germany. Archiv für Geflügelkunde 2012, 76, 234-238.

18. Park, J.Y.; Kim, S.; Oh, J.Y.; Kim, H.R.; Jang, I.; Lee, H.S.; Kwon, Y.-K. Characterization of Clostridium perfringens isolates obtained from 2010 to 2012 from chickens with necrotic enteritis in Korea. Poult. Sci. 2015, 94, 1158-1164. [CrossRef] [PubMed]

19. Watkins, K.; Shryock, T.; Dearth, R.; Saif, Y. In-vitro antimicrobial susceptibility of Clostridium perfringens from commercial turkey and broiler chicken origin. Veter Microbiol. 1997, 54, 195-200. [CrossRef]

20. Fan, Y.-C.; Wang, C.-L.; Wang, C.; Chen, T.-C.; Chou, C.-H.; Tsai, H.-J. Incidence and Antimicrobial Susceptibility to Clostridium perfringens in Premarket Broilers in Taiwan. Avian Dis. 2016, 60, 444-449. [CrossRef] [PubMed]

21. Dahiya, J.; Wilkie, D.; Van Kessel, A.; Drew, M. Potential strategies for controlling necrotic enteritis in broiler chickens in post-antibiotic era. Anim. Feed. Sci. Technol. 2006, 129, 60-88. [CrossRef]

22. Baker-Austin, C.; Wright, M.S.; Stepanauskas, R.; McArthur, J. Co-selection of antibiotic and metal resistance. Trends Microbiol. 2006, 14, 176-182. [CrossRef]

23. Andersson, D.I.; Hughes, D. Antibiotic resistance and its cost: Is it possible to reverse resistance? Nat. Rev. Genet. 2010, 8, 260-271. [CrossRef]

24. Soge, O.; Tivoli, L.; Meschke, J.; Roberts, M. A conjugative macrolide resistance gene, mef(A), in environmental Clostridium perfringens carrying multiple macrolide and/or tetracycline resistance genes. J. Appl. Microbiol. 2009, 106, 34-40. [CrossRef]

25. Abildgaard, L.; Sondergaard, T.E.; Engberg, R.; Schramm, A.; Højberg, O. In vitro production of necrotic enteritis toxin $\mathrm{B}, \mathrm{NetB}$, by netB-positive and netB-negative Clostridium perfringens originating from healthy and diseased broiler chickens. Veter Microbiol. 2010, 144, 231-235. [CrossRef] [PubMed]

26. Gu, C.; Lillehoj, H.S.; Sun, Z.; Lee, Y.; Zhao, H.; Xianyu, Z.; Yan, X.; Wang, Y.; Lin, S.; Liu, L.; et al. Characterization of Virulent netB+/tpeL+Clostridium perfringens Strains from Necrotic Enteritis-Affected Broiler Chicken Farms. Avian Dis. 2019, 63, 461-467. [CrossRef] [PubMed]

27. Da Silva, G.J.; Mendonça, N. Association between antimicrobial resistance and virulence in Escherichia coli. Virulence 2012, 3, 18-28. [CrossRef] [PubMed] 
28. Velappan, N.; Sblattero, D.; Chasteen, L.; Pavlik, P.; Bradbury, A.R. Plasmid incompatibility: More compatible than previously thought? Protein Eng. Des. Sel. 2007, 20, 309-313. [CrossRef] [PubMed]

29. Úbeda, C.; Maiques, E.; Knecht, E.; Lasa, Í.; Novick, R.P.; Penadés, J.R. Antibiotic-induced SOS response promotes horizontal dissemination of pathogenicity island-encoded virulence factors in staphylococci. Mol. Microbiol. 2005, 56, 836-844. [CrossRef]

Publisher's Note: MDPI stays neutral with regard to jurisdictional claims in published maps and institutional affiliations.

(C) 2020 by the authors. Licensee MDPI, Basel, Switzerland. This article is an open access article distributed under the terms and conditions of the Creative Commons Attribution (CC BY) license (http://creativecommons.org/licenses/by/4.0/). 